\title{
3 Research Square

\section{The effect of aerobic exercise on interoception and cognitive function in healthy university students: a non-randomized controlled trial}

\author{
Yusaku Amaya \\ Kansai Medical University \\ Tetsuya Abe ( $\nabla$ abttya@gmail.com ) \\ Kansai Medical University \\ Kenji Kanbara \\ Kansai Medical University \\ Hisaharu Shizuma \\ Kansai Medical University \\ Yasushi Akiyama \\ Kansai Medical University \\ Mikihiko Fukunaga \\ Kansai Medical University
}

\section{Research Article}

Keywords: Interoception, Aerobic exercise, Cognitive function, Heartbeat tracking task, Decision-making

Posted Date: March 11th, 2021

DOI: https://doi.org/10.21203/rs.3.rs-228812/v1

License: () (1) This work is licensed under a Creative Commons Attribution 4.0 International License. Read Full License 


\section{Abstract}

\section{Background:}

Long-term aerobic exercise is a key intervention for improving cognitive function as well as enhancing musculoskeletal and cardiorespiratory system functions. Additionally, decision-making, in which cognitive function is involved, can be an important factor for initiating and maintaining exercise. According to Damasio's somatic marker hypothesis, somatic experience plays a fundamental role in decision-making. Interoception is a sense of physiological information related to emotions and is known to influence cognitive function. Here, we investigated changes in interoception during moderate-intensity aerobic exercise at a level that could improve cognitive function.

\section{Methods:}

Healthy university students were divided into an exercise group $(n=37)$ and a control group $(n=11)$. The exercise group performed bench step exercises at an intensity of $50 \%$ heart rate reserve for 30 minutes a day, three times a week, for three months. Cognitive function was assessed by measuring auditory information/working memory processing speed with a Paced Auditory Serial Addition Task (PASAT), and interoceptive accuracy $(\mathrm{IA})$ was measured with a heartbeat tracking task.

\section{Results:}

There was a significant positive correlation between IA and PASAT scores at pre-intervention. But exercise did not lead to a significant increase in PASAT scores in comparison with the control group. IA scores were improved at 2-and 3-month post-intervention only in the exercise group.

\section{Conclusions:}

This suggested that interoception may be associated with the improvement in cognitive function produced by long-term aerobic exercise. Further investigation is warranted to clarify the causal relationship between interoception and cognitive function during exercise interventions.

\section{Trial registration:}

UMIN, UMIN000042891. 04/01/2021, retrospectively registered.

\section{Background}

Long-term aerobic exercise is expected as a key intervention for not only enhancing musculoskeletal and cardiorespiratory system functions, due to the physical load it places on the body, but also improving cognitive functions, such as memory, information processing speed (IPS), and decision-making [1]. It has been demonstrated that the basal ganglia, which are involved in movement initiation, form a network with the primary motor cortex and supplementary motor area to control subtle movements during voluntary 
movement in daily activities [2,3]. This network has been shown to be involved in higher-order functions, such as learning or cognition, and to be controlled by increases in exercise-induced neurotrophic factor release, angiogenesis, and neurogenesis [4]. These effects are, however, influenced by the condition of the individual or the exercise dose [5,6]: there are fewer sufficient studies on young or healthy people [7].

Generally, decision-making is performed using higher-order brain functions [8]. In addition, Antonio Damasio developed the somatic marker hypothesis $(\mathrm{SMH})$, whereby emotional somatic awareness of the preceding external stimulus influences decision-making through the activation of the anterior cingulate cortex (ACC), the insular cortex (IC), the ventromedial prefrontal cortex, and/or ventrolateral prefrontal cortex of the brain [9].

Interoception is a sense whereby physiological information, such as heartbeat and gastrointestinal motility, is primarily projected to the ACC and IC via the hormonal, immune, and autonomic nervous systems, resulting in perception [10]. It has been shown to be related to emotions such as anxiety $[9,11]$ and decision-making $[12,13]$. Interoception had been initially assessed only as interoceptive sensitivity (IS) using a questionnaire; and interoceptive accuracy (IA) and interoceptive awareness has been included as new assessment axes [14]. While both IS and interoceptive awareness are subjectively evaluated, IA is an objective index measured by behavioral testing.

In the field of psychosomatic medicine, alexithymia is a condition in which individuals show poor awareness of their own emotions; the consequent inability to select appropriate coping behaviors is thought to be associated with the development of psychosomatic diseases [15]. Interoception is widely considered a stable index reflective of a fixed individual trait [16,17], and lack of interoception has been suggested to be associated with alexithymia [18]. On the other hand, interoception has recently been considered a status index affected by psychological conditions $[19,20]$. While mental training improves interoception [21], the improvement of interoception relates to the reduction of symptoms in patients with somatoform disorder [22].

We hypothesized that an increase in interoception is associated with exercise-induced improvements in cognitive function through the improvement of decision-making. In this study, we aimed to identify the changes in interoception induced by exercising at a level expected to improve cognitive function in healthy university students.

\section{Methods}

\section{Subjects}

Subjects consisted of 48 university students recruited from Shijonawate Gakuen University. Student athletes and students who frequently worked out were excluded. All students had undergone health checkups, and those with a medical diagnosis of any disorder were excluded. In accordance with the study protocol approved by the ethics committee of Kansai Medical University Hospital, written informed consent was obtained from all subjects. 
The exercise group included 37 subjects, and the control 11 subjects matched for age, sex, and body mass index (BMI). Both were directed not to make an extra habit of exercising in their leisure time during the study. Four subjects in the exercise group reported difficulty in continuing with the task due to changes in their circumstances; thus, the exercise group eventually included 33 subjects.

\section{Procedure}

This study was conducted in a quiet room at Shijonawate Gakuen University between July 2018 and December 2018. The study protocol is schematically illustrated in Figure 1. In the sessions, the measurements of both cognitive function and interoception were executed in this order, and the instruction of a 30-minute step exercise was added after the measurements only for the exercise group. This session was repeated once a month, up to a total of four times. The exercise group was assigned to do the 30-minute step exercise once a day, three times a week, during the 3-month intervention. The control group was directed to spend time as usual, without any interventions. In both groups, no adverse events have occurred.

\section{Assessment}

\section{Cognitive function}

The Paced Auditory Serial Addition Task (PASAT) is an assessment tool for IPS [23]. Auditorily, 61 singledigit numbers are presented at one-second intervals, and the subjects are required to answer with the sum of two consecutive numbers. The percentages of correct answers are calculated as the score.

\section{Interoception}

We measured the IA using a heartbeat tracking task derived from the mental tracking task [24]. Although its validity remains unclear due to beliefs related to heart-rate counting [25], the high reliability of this test has been confirmed [26]. The subjects were seated and wore a wearable electrocardiograph device with electrode pads attached to their left chest (myBeat heart rate sensor, WHS-2 Union Tool Co., Ltd., Tokyo, Japan). After a 10-minute adaptation period, they counted their heart rates without taking their own pulse and with closed eyes during three different periods $(25,35$, and 45 seconds), in a random order, while electrocardiograms were recorded. Their IA scores were calculated using the following formula:

$I A=1 / 3 \Sigma[1-(\mid$ recorded count - perceived count $\mid) /$ recorded count $]$

The IA values ranged from 0 to 1, with values closer to 1 indicating a higher heartbeat tracking accuracy.

\section{Exercise intervention}

Nakayama et al. have reported a bench step exercise with a low height as an intervention to improve cognitive function [27]. The exercise has two advantages: first, the low step (20 cm high) makes the 
execution safer and easier, and second, stairs can easily be substituted for the step, making it possible to perform the exercise indoors without being influenced by the external environment. We employed the task with moderate-intensity exercise in reference to ACSM's Guidelines for Exercise Testing and Prescription [28]. The individual's target exercise intensity was set to $50 \%$ heart rate reserve (HRR) using the HRR method. The target heart rate was calculated with the formula: [(maximum heart rate - resting heart rate) $\times$ (target exercise intensity) $]+$ resting heart rate, where the maximum heart rate $=220$ - age.

During the step-exercise instruction, the examiner, a well-trained physical therapist who was not blind to the group assignment of the study, monitored the subjects' heart rates, and trained them to exercise at a consistent level of intensity to maintain a $50 \%$ HRR value. The examiner counted the steps per minute at that time, and informed subjects of the number of steps as a guide to perform the same-intensity exercise at home.

\section{Statistical analysis}

Values are presented as mean \pm SD. We used a chi-square test with Yates' correction for the comparison of sex distribution, and the $t$-test for comparison of age, BMI, resting heart rate, maximal heart rate, and the calculated 50\% HRR between the exercise and control groups. The correlation between IA and PASAT scores in the pre-intervention phase in all subjects was examined using Spearman's rank correlation coefficient. For the comparison of IA and PASAT scores between the exercise and control groups, we conducted a two-way repeated measures analysis of variance (ANOVA) with "point" (four levels: preintervention and 1,2, and 3 months post-intervention) as the within-subjects factor and "group" (two levels: the exercise and control groups) as the between-subjects factor. Subsequently, when the ANOVA results were significantly different, we used a Bonferroni correction as a multiple comparison test. Statistical analyses were performed using SPSS statistics 20.0 for Windows (SPSS Inc., Chicago, IL, USA). The a level was fixed at 0.05 .

\section{Results}

Comparison between the exercise and control groups showed no significant differences based on sex, age, BMI, resting heart rate, maximal heart rate, or the calculated $50 \%$ HRR (Table 1).

\section{Correlation between the IA and PASAT scores}

The pre-intervention IA and PASAT scores in all subjects were $0.76 \pm 0.15,65.07 \pm 14.92$, respectively $(n=$ 44). Figure 2 shows a scatter diagram with IA and PASAT scores in all subjects. Before intervention, the IA and PASAT scores showed a significant positive correlation $(r=0.347, p=0.021)$.

\section{Information processing speed}

Figure 3 represents the change in PASAT scores across the four points of the study (pre-intervention and 1,2 , and 3 months post-intervention) in the exercise and control groups. The two-way ANOVA indicated 
that the point main effects were significant $\left[F(3,126)=25.853, p<0.001, \eta^{2}=0.381\right]$. Both the pointgroup interaction $\left[F(3,126)=0.792, p=0.500, \eta^{2}=0.019\right]$ and the group main effects $[F(1,42)=2.193, p=$ $\left.0.146, \eta^{2}=0.050\right]$ were not. In the exercise group, PASAT scores were significantly higher in the 3-month post-intervention than in the pre-intervention ( $p<0.001)$, 1-month post-intervention $(p<0.001)$, or 2-month post-intervention phases $(p=0.001)$. The scores in the 2-month post-intervention were significantly higher than both in the pre-intervention $(p<0.001)$ and in the 1-month post-intervention phases $(p=0.011)$, and the 1-month post-intervention scores were significantly higher than in the pre-intervention phase $(p<$ 0.001). Meanwhile, in the control group, the PASAT scores were higher in each of the 3-month and 2month post-intervention phases than in the pre-intervention ( $p=0.001$ and 0.011 , respectively) or 1-month post-intervention phases ( $p=0.004$ and 0.015 , respectively).

\section{Interoception}

We compared the changes in IA scores over time between the exercise and control groups using a twoway ANOVA (Figure 4). There were no significant main effects of either point $[F(3,126)=0.716, p=0.515$, $\left.\eta^{2}=0.017\right]$ or group $\left[F(1,42)=0.015, p=0.903, \eta^{2}<0.001\right]$, and the point-group interaction was significant $\left[F(3,126)=3.334, p=0.032, \eta^{2}=0.074\right]$. This simple main effects test revealed that IA scores showed significant differences only in the exercise group between the pre-intervention and 2-month postintervention phases $(p=0.014)$ and between the pre-intervention and 3-month post-intervention phases ( $p$ $=0.003)$.

\section{Discussion}

In the present study, we investigated the changes in IA to learn more about the mechanism underlying the effectiveness of long-term aerobic exercise in improving cognitive function in healthy individuals. There was a significant positive correlation between IA and cognitive function before the exercise intervention. The exercise and control groups were found to exhibit different patterns of changes in their IA, and IA increased significantly after two and three months of exercise.

Our study demonstrated that interoception is a status index altered by long-term exercise intervention. Williamson et al. reported a significant increase in blood flow volume in the ACC and IC during a hand exercise task through the use of magnetic resonance imaging (MRI) and single-photon emission computed tomography [29]. Using functional MRI, Critchley et al. showed that, when attention was paid to a heartbeat tracking task, brain activation occurred not only in the ACC and IC, but also in the primary motor cortex and supplementary motor area, which govern movements [30]. These studies pointed to the existence of a neurological network in the regions that govern movement and interoception in the central nervous system; our findings have, as such, provided additional evidence of this link.

Interoception may be the key element for an appropriate execution of physical exercise. Our results support previous reports that have demonstrated the association between interoception and cognitive function [30]. During decision-making, information transmitted from inside and outside of the body is 
collected in the frontal lobe, and is integrated with accumulated memory. Fully organized information is re-transmitted to the brain cortex region that plays a role in the initiation of movement [31,32]. Interoception is assumed to be involved in both of these processes, and an association between high IA scores and suitable decision-making has been reported [12,13]. Moreover, some studies have used pedaling a cycle ergometer (for adults [33]) and 6-minute running (for children [34]) to confirm that subjects with high IA scores can adjust their amounts of physical activity more appropriately than those with low IA scores. These previous studies suggest that the association between interoception and decision-making will influence the effects of exercise.

In this research, we chose $50 \%$ HRR exercise, an intensity classified as moderate, as a physical intervention. High-intensity exercise has been shown to improve both cognitive and mental functions $[35,36]$. Brain-derived neurotrophic factor (BDNF), a neuroendocrine biomarker that relates to improve cognitive function, has been reported to increase after high-intensity exercise [37].

In recent years, moderate-intensity exercise has been also confirmed to improve cognitive and mental function [27,38], and it has been demonstrated that neurogenesis and neuroendocrine responses can be induced by not only high-intensity exercise but also moderate-intensity exercise [39,40]. Also in healthy young subjects, moderate-intensity exercise has been well assessed as an intervention to improve cognitive function [39-42].

Moreover, exercise enjoyment has been reported to improve through moderate-intensity exercise, but not through high-intensity exercise [43]. Steptoe and Bolton [44] have shown that high-intensity exercise did not diminish anxiety. The difficulties in initiating and maintaining exercise therapy remain a significant concern, and individuals who dropped out showed lower baseline exercise enjoyment [43]. This points to an association between mood and exercise maintenance. While the existence of neurological networks where high-intensity exercise improves cognitive function have been demonstrated, another network associated with the link between emotion and decision-making, known as the $\mathrm{SMH}$, has been examined. Moderate-intensity exercise is estimated to stimulate both networks to improve cognitive function. Emotion has been shown to correlate with interoception [9,11], and these suggest that interoception can increase more significantly in moderate-intensity exercise than in high-intensity exercise.

Our results pointed to the effectiveness of physical intervention for improving IA. Based on previous findings, therapeutic interventions with a focus on improving interoception have been investigated in clinical settings. Our study used a 30-minute step exercise three times a week for 12 weeks. Bornemann and Singer reported improvement in IA at 24 weeks using meditative psycho-training, involving 30 minutes of mindfulness practice every day [45]. Mehling et al. used integrated training involving 50 minutes of yoga exercises or several types of resistance training, three times a week for 12 weeks, as well as conventional mindfulness training [46]. These studies clinically indicate that several types of interventions, such as psychotherapy and physical training, can improve interoception, and that even an independent intervention consisting of aerobic exercise can be effective for it as well. Moderate-intensity physical interventions, rather than psychological interventions, may effectively affect interoception within 
a shorter period, and the exercise load for interventions may not necessarily need to be high. Additionally, mindfulness, a self-training psychotherapy aimed at making emotions conscious, is effective not only in minimizing negative mood [47], but also in increasing working memory [48]. Neuroimaging studies have also suggested that mindfulness is associated with the ACC, IC, temporoparietal junction, and limbic network [49]. These studies demonstrate the potential of the concomitant use of psychotherapy, such as mindfulness training, with physical interventions to accelerate exercise-induced improvements in cognitive function through changes in interoception.

\section{Limitations}

First, while our results demonstrated a cross-sectional correlation between interoception and cognitive function, as previously reported [30], we could not identify a causal relationship. The main limitation involved the assessment of cognitive function. Indeed, the repetitive use of PASAT is the first main limitation. While PASAT is an established tool to measure IPS, practice effects have been also reported [50]. The other limitation comprised the subjects' characteristics. PASAT was originally developed for patients with neurological dysfunction. Our subjects were healthy, young, and intelligent individuals, and under cognitive stimulation; each of these factors can independently increase IPS [51,52], and may potentially lead to the ceiling effect. These factors might obscure results linked to the PASAT changes induced by exercise intervention. However, some studies have also employed PASAT in healthy young subjects $[53,54]$. The PASAT reference value for healthy Japanese individuals in their twenties is $57.7 \pm$ 14.71 [55], which is similar to that reported here. Otherwise, it is possible that three months of exercise was insufficient to improve cognitive function.

Next, there is no objective data representing the subjects' activities either in their daily lives or during exercise intervention. However, we chose subjects whose base activities were expected to be low to best elucidate the effects of the intervention.

Last, although the present study included subjects of both sexes, several studies have reported sex differences in interoception [56]. Verification of our findings across a wider age range, for both sexes individually, and with an increased sample size of the general population, is warranted.

\section{Conclusions}

Our study found improved interoception, which may increase cognitive function, after long-term moderate-intensity aerobic exercise, and a significant correlation between interoception and cognitive function before the intervention. Although we failed to effectively demonstrate the increase in cognitive function induced by exercise, our findings point towards an association between interoception and the effectiveness of aerobic exercise in improving cognitive function.

\section{Declarations}

\section{Ethics approval and consent to participate}


All procedures performed in studies involving human participants were in accordance with the ethical committee of Kansai Medical University (approval number: 2017268) and with the 1964 Helsinki declaration and its later amendments or comparable ethical standards. All participants were above 18 years of age, and provided their written informed consent.

\section{Consent for publication}

Not applicable.

\section{Availability of data and materials}

Data of participants who agreed to the public distribution of data are available from the corresponding author upon reasonable request.

\section{Competing interests}

The authors declare that they have no competing interests.

\section{Funding}

This study received no funding.

\section{Authors' contributions}

YA, TA, KK, and MF devised and designed the study. YA conducted the experiment. All authors contributed to data analysis. YA, HS, and YA provided resources such as research equipment. YA and TA wrote and compiled the manuscript. TA, KK, YA, and MF provided advice and guidance throughout the study. All authors read and approved the final manuscript.

\section{Acknowledgements}

We would like to thank Editage (www.editage.com) and Silver Academic Editing (www.silverediting.com) for English language editing.

\section{Abbreviations}

ACC: anterior cingulate cortex

BMI: body mass index

BDNF: brain-derived neurotrophic factor

CAT: clinical assessment for attention

HRR: heart rate reserve 
IA: interoceptive accuracy

IC: insular cortex

IPS: information processing speed

IS: interoceptive sensitivity

MRI: magnetic resonance imaging

PASAT: paced auditory serial addition task

SMH: somatic marker hypothesis

\section{References}

1. Erickson Kl, Hillman C, Stillman CM, Ballard RM, Bloodgood B, Conroy DE, et al. Physical Activity, Cognition, and Brain Outcomes: A Review of the 2018 Physical Activity Guidelines. Med Sci Sports Exerc. 2019;51(6):1242-51.

2. Alexander GE, Crutcher MD. Functional architecture of basal ganglia circuits: neural substrates of parallel processing. Trends Neurosci. 1990;13(7):266-71.

3. Takakusaki K. Functional Neuroanatomy for Posture and Gait Control. J Mov Disord. 2017;10(1):117.

4. Leisman G, Braun-Benjamin O, Melillo R. Cognitive-motor interactions of the basal ganglia in development. Front Syst Neurosci. 2014;8:16.

5. Crush EA, Loprinzi PD. Dose-Response Effects of Exercise Duration and Recovery on Cognitive Functioning. Percept Mot Skills. 2017;124(6):1164-93.

6. Cabral DF, Rice J, Morris TP, Rundek T, Pascual-Leone A, Gomes-Osman J. Exercise for Brain Health: An Investigation into the Underlying Mechanisms Guided by Dose. Neurotherapeutics. 2019;16(3):580-99.

7. Lubans D, Richards J, Hillman C, Faulkner G, Beauchamp M, Nilsson M, et al. Physical activity for cognitive and mental health in youth: A systematic review of mechanisms. Pediatrics. 2016;138(3):e20161642.

8. Qiu L, Su J, Ni Y, Bai Y, Zhang X, Li X, et al. The neural system of metacognition accompanying decision-making in the prefrontal cortex. PLoS Biol. 2018;16(4):e2004037.

9. Damasio AR. Descartes' error: emotion, reason, and the human brain. G.P. Putnam; 1994.

10. Craig AD. Interoception: The sense of the physiological condition of the body. Curr Opin Neurobiol. 2003;13(4):500-5.

11. Terasawa $Y$, Fukushima $H$, Umeda $S$. How does interoceptive awareness interact with the subjective experience of emotion? An fMRI Study. Hum Brain Mapp. 2013 Mar;34(3):598-612. 
12. Kandasamy N, Garfinkel SN, Page L, Hardy B, Critchley HD, Gurnell M, et al. Interoceptive Ability Predicts Survival on a London Trading Floor. Sci Rep. 2016;6:32986.

13. Werner NS, Jung K, Duschek S, Schandry R. Enhanced cardiac perception is associated with benefits in decision-making. Psychophysiology. 2009;46(6):1123-9.

14. Garfinkel SN, Tiley C, O’Keeffe S, Harrison NA, Seth AK, Critchley HD. Discrepancies between dimensions of interoception in autism: Implications for emotion and anxiety. Biol Psychol. 2016;114:117-26.

15. Sifneos PE. The prevalence of "Alexithymic" characteristics in psychosomatic patients. Psychother Psychosom. 1973;22(2-6):255-62.

16. Ehlers A, Breuer P. How good are patients with panic disorder at perceiving their heartbeats? Biol Psychol. 1996;42(1-2):165-82.

17. Stevens S, Gerlach AL, Cludius B, Silkens A, Craske MG, Hermann C. Heartbeat perception in social anxiety before and during speech anticipation. Behav Res Ther. 2011;49(2):138-43.

18. Murphy J, Brewer R, Hobson H, Catmur C, Bird G. Is alexithymia characterised by impaired interoception? Further evidence, the importance of control variables, and the problems with the Heartbeat Counting Task. Biol Psychol. 2018;136:189-97.

19. Herbert BM, Pollatos O, Flor H, Enck P, Schandry R. Cardiac awareness and autonomic cardiac reactivity during emotional picture viewing and mental stress. Psychophysiology. 2010;47(2):34254.

20. Fairclough SH, Goodwin L. The effect of psychological stress and relaxation on interoceptive accuracy: Implications for symptom perception. J Psychosom Res. 2007;62(3):289-95.

21. Bornemann B, Singer T. Taking time to feel our body: Steady increases in heartbeat perception accuracy and decreases in alexithymia over 9 months of contemplative mental training. Psychophysiology. 2017;54(3):469-82.

22. Schaefer M, Egloff B, Gerlach AL, Witthöft M. Improving heartbeat perception in patients with medically unexplained symptoms reduces symptom distress. Biol Psychol. 2014;101:69-76.

23. Gronwall DMA. Paced auditory serial addition task: A measure of recovery from concussion. Percept Mot Skills. 1977;44(2):367-73.

24. Schandry R. Heart Beat Perception and Emotional Experience. Psychophysiology. 1981;18(4):483-8.

25. Ring C, Brener J, Knapp K, Mailloux J. Effects of heartbeat feedback on beliefs about heart rate and heartbeat counting: A cautionary tale about interoceptive awareness. Biol Psychol. 2015;104:193-8.

26. Kleckner IR, Wormwood JB, Simmons WK, Barrett LF, Quigley KS. Methodological recommendations for a heartbeat detection-based measure of interoceptive sensitivity. Psychophysiology. 2015;52(11):1432-40.

27. Nakayama F, Tobina T, Ayabe M, Doi Y, Mori Y, Yamada T, et al. Home based exercise effects on cognition in the semi-independent elderly. Jpn J Phys Fit Sport Med. 2011;60(4):379-86. 
28. American College of Sports Medicine. ACSM's Guidelines for Exercise Testing and Prescription. Lippincott Williams \& Wilkins; 2017.

29. Williamson JW, McColl R, Mathews D. Evidence for central command activation of the human insular cortex during exercise. J Appl Physiol. 2003;94(5):1726-34.

30. Critchley HD, Wiens S, Rotshtein P, Öhman A, Dolan RJ. Neural systems supporting interoceptive awareness. Nat Neurosci. 2004;7(2):189-95.

31. Middleton FA, Strick PL. Basal ganglia output and cognition: Evidence from anatomical, behavioral, and clinical studies. Brain Cogn. 2000;42(2):183-200.

32. Takakusaki K, Saitoh K, Harada H, Kashiwayanagi M. Role of basal ganglia-brainstem pathways in the control of motor behaviors. Neurosci Res. 2004;50(2):137-51.

33. Herbert BM, Ulbrich P, Schandry R. Interoceptive sensitivity and physical effort: Implications for the self-control of physical load in everyday life. Psychophysiology. 2007;44(2):194-202.

34. Georgiou E, Matthias E, Kobel S, Kettner S, Dreyhaupt J, Steinacker JM, et al. Interaction of physical activity and interoception in children. Front Psychol. 2015;6:1-8.

35. Erickson KI, Voss MW, Prakash RS, Basak C, Szabo A, Chaddock L, et al. Exercise training increases size of hippocampus and improves memory. Proc Natl Acad Sci U S A. 2011;108(7):3017-22.

36. Knubben K, Reischies FM, Adli M, Schlattmann P, Bauer M, Dimeo F. A randomised, controlled study on the effects of a short-term endurance training programme in patients with major depression. $\mathrm{Br} J$ Sports Med. 2007;41(1):29-33.

37. Ferris LT, Williams JS, Shen CL. The effect of acute exercise on serum brain-derived neurotrophic factor levels and cognitive function. Med Sci Sports Exerc. 2007;39(4):728-34.

38. Inoue DS, Monteiro PA, Gerosa-Neto J, Santana PR, Peres FP, Edwards KM, et al. Acute increases in brain-derived neurotrophic factor following high or moderate-intensity exercise is accompanied with better cognition performance in obese adults. Sci Rep. 2020;10(1):13493.

39. Zoladz JA, Pilc A, Majerczak J, Grandys M, Zapart-Bukowska J, Duda K. Endurance training increases plasma brain-derived neurotrophic factor concentration in young healthy men. J Physiol Pharmacol. 2008;59 Suppl 7:119-32.

40. Suwabe K, Hyodo K, Byun K, Ochi G, Fukuie T, Shimizu T, et al. Aerobic fitness associates with mnemonic discrimination as a mediator of physical activity effects: Evidence for memory flexibility in young adults. Sci Rep. 2017;7(1):5140.

41. Hung C-L, Tseng J-W, Chao H-H, Hung T-M, Wang H-S. Effect of Acute Exercise Mode on Serum BrainDerived Neurotrophic Factor (BDNF) and Task Switching Performance. J Clin Med. 2018;7(10):301.

42. Stern Y, Mackay-Brandt A, Lee S, McKinley P, Mclntyre K, Razlighi Q, et al. Effect of aerobic exercise on cognition in younger adults: A randomized clinical trial. Neurology. 2019;92(9):E905-16.

43. Heinrich KM, Patel PM, O’Neal JL, Heinrich BS. High-intensity compared to moderate-intensity training for exercise initiation, enjoyment, adherence, and intentions: An intervention study. BMC Public Health. 2014;14(1):789. 
44. Steptoe A, Bolton J. The Short-term influence of high and low intensity physical exercise on mood. Psychol Health. 1988;2(2):91-106.

45. Bornemann B, Singer T. Taking time to feel our body: Steady increases in heartbeat perception accuracy and decreases in alexithymia over 9 months of contemplative mental training. Psychophysiology. 2017;54(3):469-82.

46. Mehling WE, Chesney MA, Metzler TJ, Goldstein LA, Maguen S, Geronimo C, et al. A 12-week integrative exercise program improves self-reported mindfulness and interoceptive awareness in war veterans with posttraumatic stress symptoms. J Clin Psychol. 2018;74(4):554-65.

47. van der Zwan JE, de Vente W, Huizink AC, Bögels SM, de Bruin El. Physical Activity, Mindfulness Meditation, or Heart Rate Variability Biofeedback for Stress Reduction: A Randomized Controlled Trial. Appl Psychophysiol Biofeedback. 2015;40(4):257-68.

48. Quach D, Jastrowski Mano KE, Alexander K. A Randomized Controlled Trial Examining the Effect of Mindfulness Meditation on Working Memory Capacity in Adolescents. J Adolesc Health. 2016;58(5):489-96.

49. Hölzel BK, Lazar SW, Gard T, Schuman-Olivier Z, Vago DR, Ott U. How does mindfulness meditation work? Proposing mechanisms of action from a conceptual and neural perspective. Perspect Psychol Sci. 2011;6(6):537-59.

50. Tombaugh TN. A comprehensive review of the Paced Auditory Serial Addition Test (PASAT). Arch Clin Neuropsychol. 2006;21(1):53-76.

51. Scherr M, Kunz A, Doll A, Mutzenbach JS, Broussalis E, Bergmann HJ, et al. Ignoring floor and ceiling effects may underestimate the effect of carotid artery stenting on cognitive performance. $J$ Neurointerv Surg. 2016;8(7):747-51.

52. Karssemeijer EGA, Aaronson JA, Bossers WJR, Donders R, Olde Rikkert MGM, Kessels RPC. The quest for synergy between physical exercise and cognitive stimulation via exergaming in people with dementia: A randomized controlled trial 17 Psychology and Cognitive Sciences 1701 Psychology 11 Medical and Health Sciences 1103 Clinical Sciences. Alzheimer's Res Ther. 2019;11(1):3.

53. Tanosoto T, Bendixen KH, Arima T, Hansen J, Terkelsen AJ, Svensson P. Effects of the paced auditory serial addition task (PASAT) with different rates on autonomic nervous system responses and selfreported levels of stress. J Oral Rehabil. 2015;42(5):378-85.

54. Mathias CW, Stanford MS, Houston RJ. The physiological experience of the Paced Auditory Serial Addition Task (PASAT): Does the PASAT induce autonomic arousal? Arch Clin Neuropsychol. 2004;19(4):543-54.

55. Japan Society for Higher Brain Dysfunction. The manual for clinical assessment for attention (CAT) and clinical assessment for spontaneity (CAS). Shinko Igaku Shuppan Tokyo; 2006.

56. Harver A, Katkin ES, Bloch E. Signal-detection outcomes on heartbeat and respiratory resistance detection tasks in male and female subjects. Psychophysiology. 1993;30(3):223-30.

\section{Table}


Table 1. Demographic data of subjects

\begin{tabular}{cccc}
\hline & Exercise & Control & P value \\
& $(\mathrm{N}=33)$ & $(\mathrm{N}=11)$ & \\
\hline Female (\%) & $9(27.3 \%)$ & $5(45.5 \%)$ & 0.29 \\
Age & & & \\
(mean \pm SD, years) & $21.1 \pm 1.1$ & $21.4 \pm 0.5$ & 0.45 \\
BMI & & & \\
(mean \pm SD, kg/m $\left.{ }^{2}\right)$ & $22.4 \pm 3.2$ & $22.3 \pm 3.6$ & 0.95 \\
\hline HR-max & & & \\
(mean \pm SD, bpm) & $198.89 \pm 0.98$ & $198.64 \pm 0.48$ & 0.42 \\
\hline HR-rest & & & \\
$($ mean \pm SD, bpm) & $69.51 \pm 9.36$ & $73.27 \pm 8.97$ & 0.25 \\
\hline 50\% HRR & & & \\
\hline (mean \pm SD, bpm) & $134.07 \pm 5.53$ & $137.23 \pm 8.32$ & 0.15 \\
\hline
\end{tabular}

$\mathrm{N}=$ Number; $\mathrm{SD}=$ Standard Deviation; $\mathrm{BMI}=$ Body Mass Index. HR $=$ Heart Rate; HR-rest $=$ resting Heart Rate; HR-max = maximal Heart Rate; HRR = Heart Rate Reserve.

Figures

Exercise group $(\mathrm{N}=33)$

Step exercise [50\% HRR] (30 minutes a day, 3 times a week)

Control group $(\mathrm{N}=11)$ (No exercise intervention)

Session

PASAT

IA

Step exercise (Exercise group only)

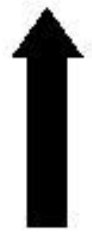

Preintervention

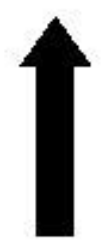

$1 \mathrm{M}$

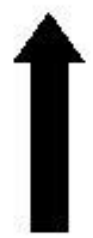

$2 \mathrm{M}$

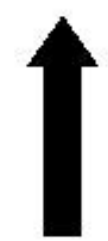

$3 \mathrm{M}$

Post-intervention

\section{Figure 1}


Study protocol The session consists of the PASAT and IA measurements and the step exercise training in this order. Both the exercise and control groups executed the sessions at pre-intervention and 1-, 2-, and 3month post-intervention. $\mathrm{N}=$ Number; HRR = Heart Rate Reserve; PASAT = Paced Auditory Serial Addition Task; IA = Interoceptive Accuracy; $\mathrm{M}$ = Month.

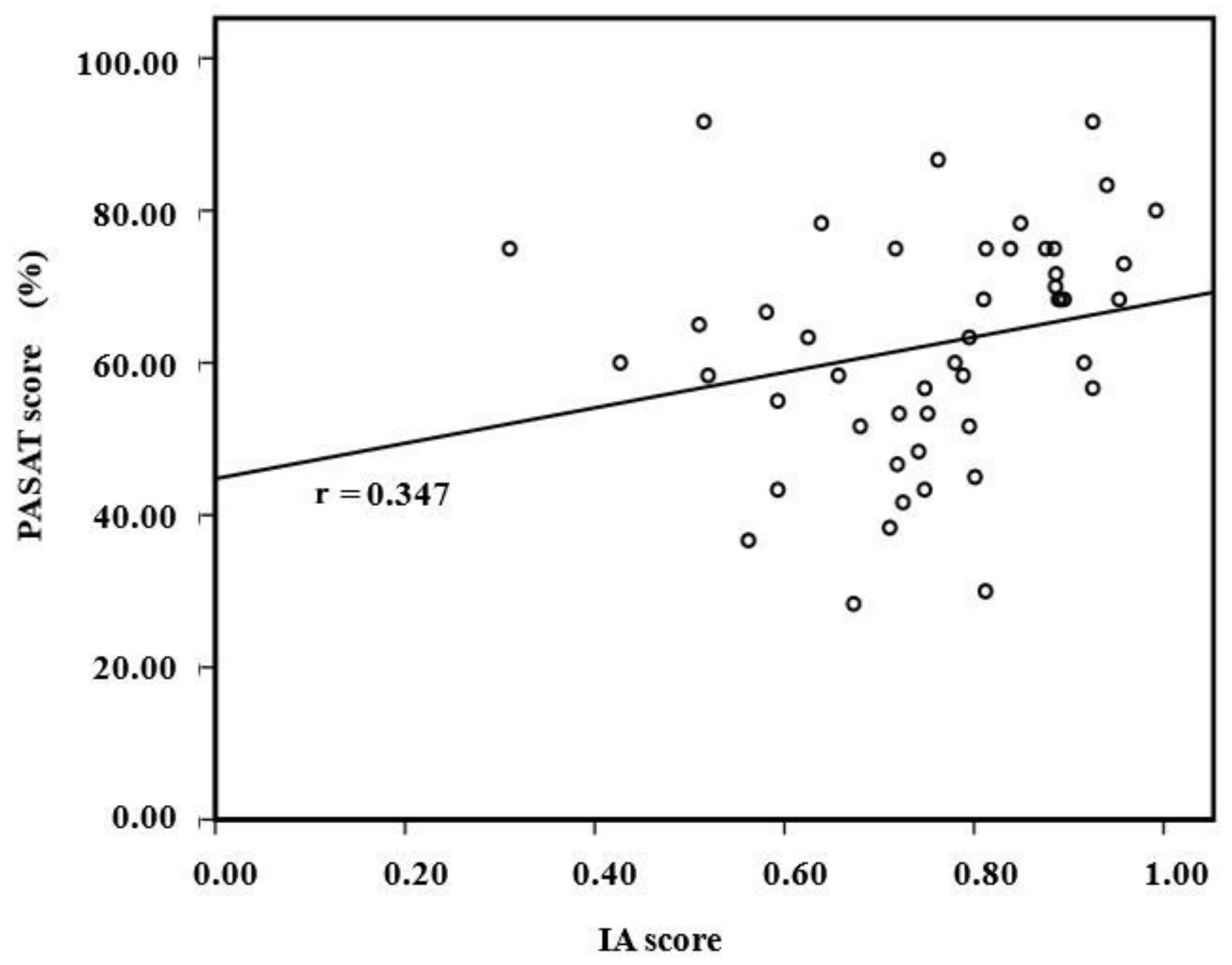

Figure 2

Correlation between IA and PASAT in all subjects pre-intervention IA = Interoceptive Accuracy; PASAT = Paced Auditory Serial Addition Task. 


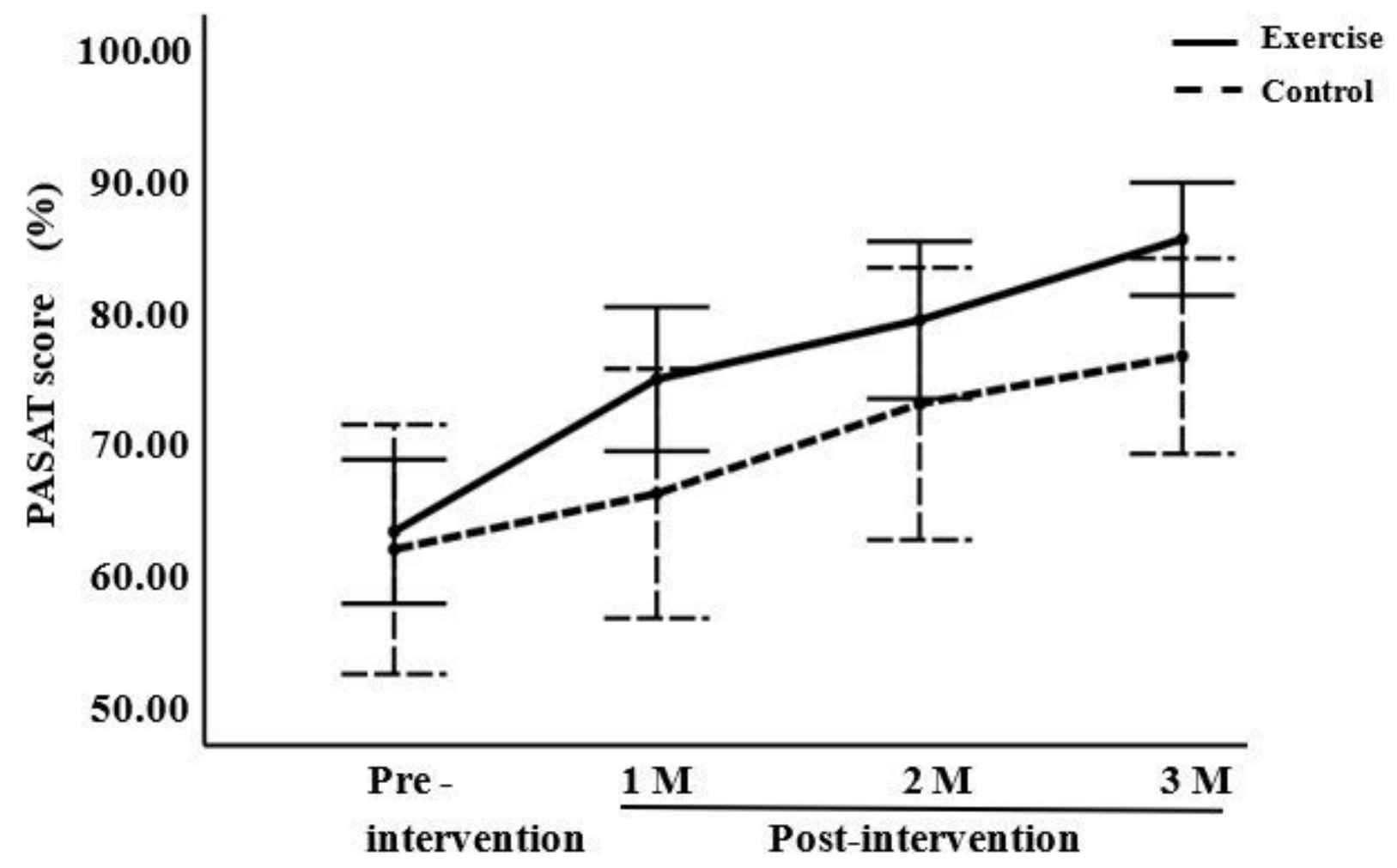

Figure 3

PASAT change induced by exercise in the exercise and control groups PASAT = Paced Auditory Serial Addition Task; $\mathrm{M}=$ Month. 


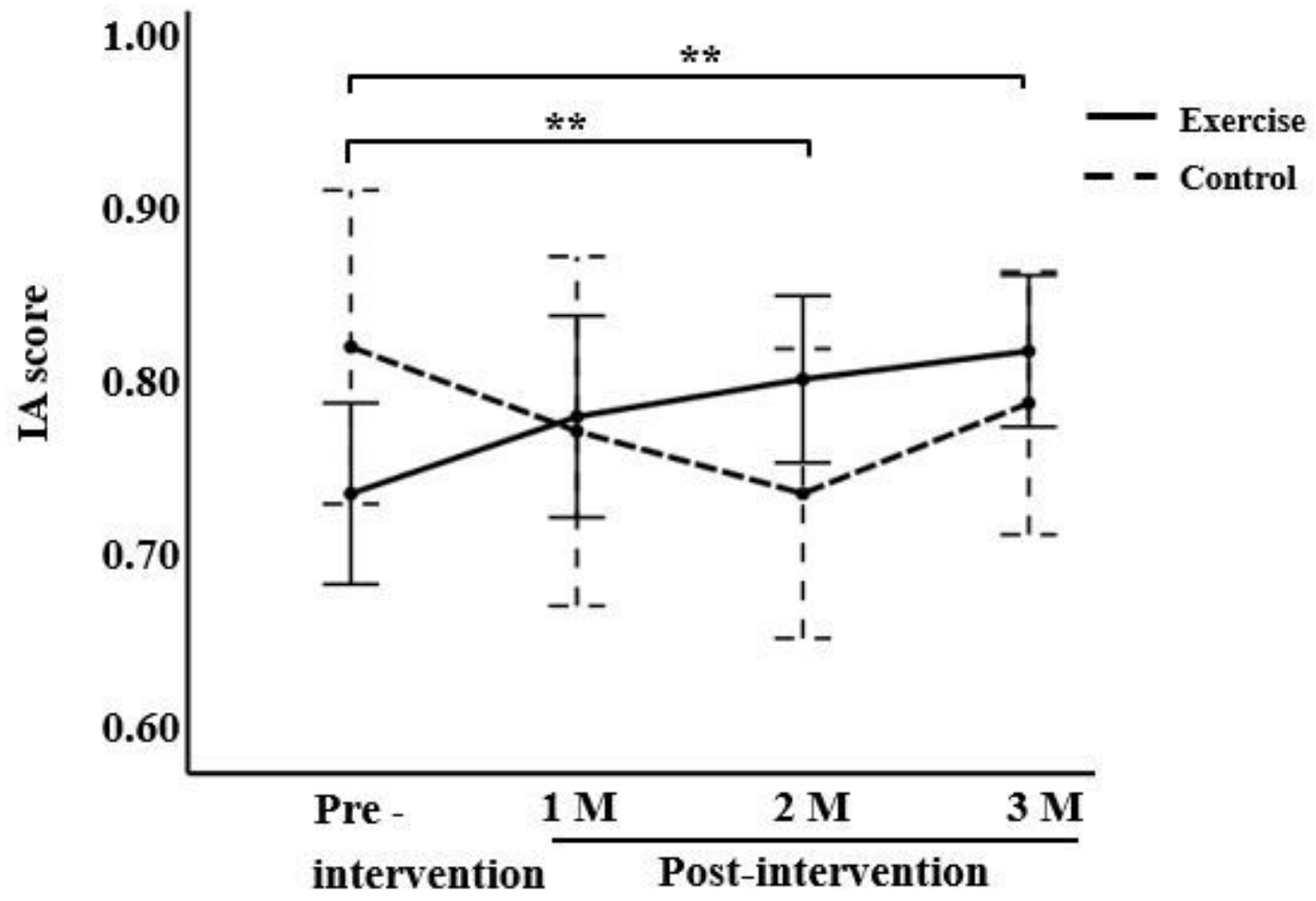

\section{Figure 4}

IA change induced by exercise in the exercise and control groups $\mathrm{I} A=$ Interoceptive Accuracy; $\mathrm{M}=$ Month . ** $p<0.01$ (compared in the exercise group). 